\title{
Use of different bioreactor types for citric acid production by Yarrowia lipolytica
}

Patrícia Ferreira*, Ana S. Pereira, Isabel Belo

Centre of Biological Engineering, University of Minho, Braga, Portugal

E-mail address: patricia.ferreira@ceb.uminho.pt (P. Ferreira).

Yarrowia lipolytica, a strictly aerobic yeast, can produce several added-value products from different carbon sources, including some agro-industrial wastes. Citric acid, extensively used in many industries, can be produced by $Y$. lipolytica from crude glycerol. Being this an aerobic bioprocess, the oxygen concentration is a key factor in the citric acid production. Thus, a main challenge on bioreactor selection and operation is to provide an adequate oxygen mass transfer rate (OTR) from the inlet gas into the culture medium. Citric acid production by $Y$. lipolytica from glycerol was accessed using three different bioreactors: a pressurized, an airlift bioreactor and a stirred tank reactor (STR). Increased OTR's were obtained by raising the total air pressure in the pressurized bioreactor, increasing the specific air flow rate in the airlift bioreactor and varying the aeration and agitation rate in the STR bioreactor. An improvement of $40 \%$ in citric acid concentration was obtained raising the air pressure up to 2 bar in the pressurized bioreactor, whereas in the airlift bioreactor, a 30\% improvement was attained by $50 \%$ increase of specific air flow rate and an increase of 7.7 -fold was observed raising OTR from 52 to $408 \mathrm{mg} \mathrm{L}^{-1} \mathrm{~h}^{-1}$ using the STR. 